\title{
A multi-spectral fluorescence induction and relaxation (FIRe) technique for physiological and taxonomic analysis of phytoplankton communities
}

\author{
Maxim Y. Gorbunov ${ }^{1, *}$, Evgeny Shirsin ${ }^{2}$, Elena Nikonova ${ }^{2}$, Victor V. Fadeev ${ }^{2}$, \\ Paul G. Falkowski ${ }^{1,3}$ \\ ${ }^{1}$ Environmental Biology and Molecular Ecology Program, Department of Marine and Coastal Sciences, Rutgers, \\ the State University of New Jersey, New Brunswick, New Jersey 08901, USA \\ ${ }^{2}$ Physics Department, MV Lomonosov Moscow State University, Moscow, 119991, Russia \\ ${ }^{3}$ Department of Earth and Planetary Sciences, Rutgers, the State University of New Jersey, Piscataway, New Jersey 08854, USA
}

\begin{abstract}
Phytoplankton are extraordinarily diverse, comprising 13 phylogenetic groups, with diatoms, dinoflagellates, and haptophytes among the most prominent eukaryotes in the ocean. Development of sensor technologies for rapid taxonomic and physiological analysis of phytoplankton communities is crucial for ecological monitoring programs in the global ocean. We describe a novel, ultra-sensitive, multi-spectral fluorescence induction and relaxation instrument (a mini-FIRe) and examine its analytical capability of rapidly determining phytoplankton taxonomic groups, as well as physiological characteristics and photosynthetic rates. We collected and analyzed the database of spectral and photosynthetic properties of major taxonomic groups of phytoplankton. We revealed that the spectral shape of the functional absorption cross-section of Photosystem II (PSII), $\sigma_{\text {PSII }}\left(\lambda_{\text {ex }}\right)$, is remarkably constrained within each major phylogenetic group of eukaryotic phytoplankton, including diatoms, haptophytes, dinoflagellates, and chlorophytes. Variability in $\sigma_{\mathrm{PSII}}\left(\lambda_{\mathrm{ex}}\right)$ within each group was significantly smaller than the difference between groups. We also examined the classical excitation spectra of chl a fluorescence yields, $F_{\mathrm{m}}\left(\lambda_{\mathrm{ex}}\right)$. Our comparative analysis revealed that $\sigma_{\mathrm{PSII}}\left(\lambda_{\mathrm{ex}}\right)$ is a better and more specific proxy for taxonomic analysis. For instance, our developed $\sigma_{\mathrm{PSII}}$-based algorithm correctly identified $90 \%$ of experimental data, compared to $77 \%$ identified by the $F_{\mathrm{m}}$-based algorithm. Our results suggest that the multi-color variable fluorescence analysis offers a tool for combined physiological and taxonomic analysis, including identification of major phyla within the 'red' lineage of eukaryotic phytoplankton.
\end{abstract}

*Corresponding author: gorbunov@marine.rutgers.edu

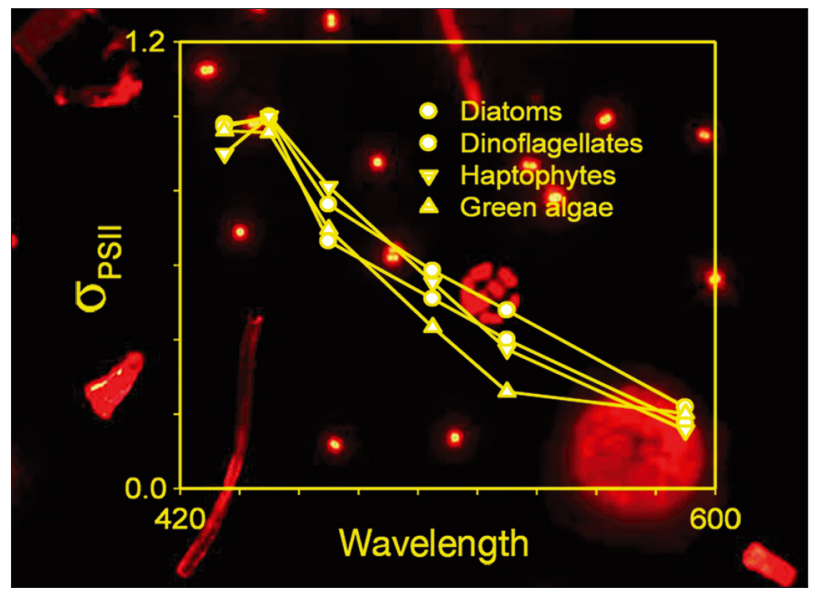

Phytoplankton cells emit red fluorescence from chlorophyll with the fluorescence quantum yield reflecting physiological status and the spectral properties (graph of $\sigma_{\text {PSII }}$, r.u. against wavelength, $\mathrm{nm}$ ) reflecting taxonomic identity.

Photo: M. Gorbunov

KEY WORDS: Variable fluorescence $\cdot$ Photophysiology $\cdot$ Photosynthesis $\cdot$ Phytoplankton composition

\section{INTRODUCTION}

Phytoplankton photosynthesis in the ocean accounts for approximately half of the global carbon fixation on Earth (Field et al. 1998). Unlike terrestrial plants, however, phytoplankton are phylogenetically diverse, comprising 3 basic evolutionary lineages

(C) The authors 2020. Open Access under Creative Commons by Attribution Licence. Use, distribution and reproduction are unrestricted. Authors and original publication must be credited. 
(Delwiche 1999). The first lineage contains all prokaryotic oxygenic organisms, cyanobacteria, that presumably evolved more than 2.8 billion yr ago and played a fundamental role in forming oxygen, carbon, and nitrogen cycles on the planet. All other oxygen-evolving organisms are eukaryotic and contain 13 phyla with more than 20000 species (Falkowski et al. 2004a). One group of eukaryotes uses chlorophyll $b$ as a secondary photosynthetic pigment and forms the 'green lineage' from which all terrestrial plants descended. The 'green lineage' played a key role in the ocean from ca. 700 million until 250 million yr ago (Falkowski et al. 2004a). Since that time, another group of eukaryotes, called the 'red lineage,' radiated rapidly and became ecologically dominant in the modern ocean (Falkowski et al. 2004a). The 'red lineage' of phytoplankton comprises 12 phyla, including diatoms, dinoflagellates, and haptophytes, which are the most ecologically prominent (Falkowski \& Raven 2007). These 3 'red' taxonomic groups use chlorophyll $c$ and a series of taxonspecific carotenoids as secondary pigments. Species within the 'red' lineage can form massive phytoplankton blooms in coastal, sub-polar, and polar regions of the modern ocean and are responsible for most carbon fixation and sequestration into the ocean interior.

In natural aquatic ecosystems, phytoplankton community structure and physiology vary both horizontally and vertically on micro-, meso-, and regional spatial scales. Community structure is commonly analyzed using microscopic and/or pigment analyses (Claustre 1994, Mackey et al. 1996, Bidigare et al. 2005, Uitz et al. 2006, 2008, Sathyendranath et al. 2009) or flow cytometry (Chisholm et al. 1988, Olson et al. 1991). While conventional flow cytometers are primarily designed for and largely limited to the analysis of pico- and nano-plankton (Chisholm et al. 1988, Li 1997, Olson et al. 2003), imaging-in-flow systems target larger cells (Sieracki et al. 1998, Davis et al. 2004, 2005, Olson \& Sosik 2007, Sosik \& Olson 2007). While all of these approaches have the advantage of precision and accuracy, they are invariably time consuming and labor intensive. Sample throughput becomes a limiting factor to determining patterns of distributions of photosynthetic organisms and rates.

Major functional groups of phytoplankton primarily differ and are characterized by their pigment composition and, as a result, by their spectral properties. Following pioneering works (Yentsch \& Yentsch 1979, Yentsch \& Phinney 1984), spectralresolved measurements of fluorescence have been proposed and explored as a potential tool for rapid monitoring of variations in community structure (Oldham \& Warner 1987, Seppälä \& Balode 1997, Babin et al. 2005, MacIntyre et al. 2010, Richardson et al. 2010). Because spectral analysis requires very high signal-to-noise ratios and precision, the previously developed instruments have a lower limit of sensitivity of only $\sim 0.5$ to $1.0 \mathrm{mg}$ chlorophyll m $\mathrm{m}^{-3}$ that precludes their applications in the open ocean. Also, although time series of flow-cytometric sampling potentially allow one to deduce growth rates (Sosik et al. 2003), none of the above techniques provides information about phytoplankton physiology or photosynthetic rates.

Variable fluorescence techniques, such as fast repetition rate (FRR) fluorometry (Kolber et al. 1998) and fluorescence induction and relaxation (FIRe) technique (Gorbunov \& Falkowski 2005) have been developed for in situ measurements of phytoplankton biomass and photophysiology in the ocean. These techniques are based on recording, with microsecond time resolution, fluorescent transients induced by a precisely controlled sequence of excitation flashes of light. The induced 'variable fluorescence' signals are driven by variations in the physiological status of the photosynthetic apparatus and provide a comprehensive suite of photosynthetic properties of light harvesting, photochemistry in Photosystem II (PSII), and electron transport down to carbon fixation. This analysis provides the basis for stress diagnostic protocols (Falkowski et al. 2004b). The use of FRR and FIRe instruments has provided unprecedented information about the functioning of phytoplankton communities and the impact of environmental factors controlling primary productivity in the ocean (e.g. Behrenfeld et al. 1996, Coale et al. 2004, Falkowski et al. 2004b, Lin et al. 2016) and is becoming increasingly important for most biological oceanographic programs.

In an effort to expand capabilities of variable fluorescence for taxonomic analysis, we examined the combination of variable fluorescence and spectral analyses that offers a potential for simultaneous determination of biomass, photophysiology, and taxonomy in natural aquatic ecosystems.

In this paper, we describe a novel ultra-sensitive multispectral FIRe instrument (a mini-FIRe) and examine analytical capabilities of this technique for rapid determination of phytoplankton functional groups, together with physiological characteristics and photosynthetic rates. For this goal, we examined the spectral dependences of both 
fluorescence intensities $\left(F_{\mathrm{m}}\right)$ and functional absorption cross-sections of PSII $\left(\sigma_{\mathrm{PSII}}\right)$, to reconstruct the basic spectra of each major phylogenetic group of eukaryotes. Model experiments were performed with a set of 25 cultures in a wide range of environmental conditions (illumination and nutrient limitation). Using this framework, we developed and tested algorithms for taxonomic identification based on the spectral properties of $\sigma_{\text {PSII }}\left(\lambda_{\text {ex }}\right)$, where $\lambda_{\text {ex }}$ is the wavelength of excitation light. This approach was compared to conventional analysis of fluorescence excitation spectra. Our results revealed that the spectral properties of functional cross-sections $\left(\sigma_{\mathrm{PSII}}\right)$ are highly conserved for each functional group and can serve as a better taxonomic indicator than classical fluorescence excitation spectra.

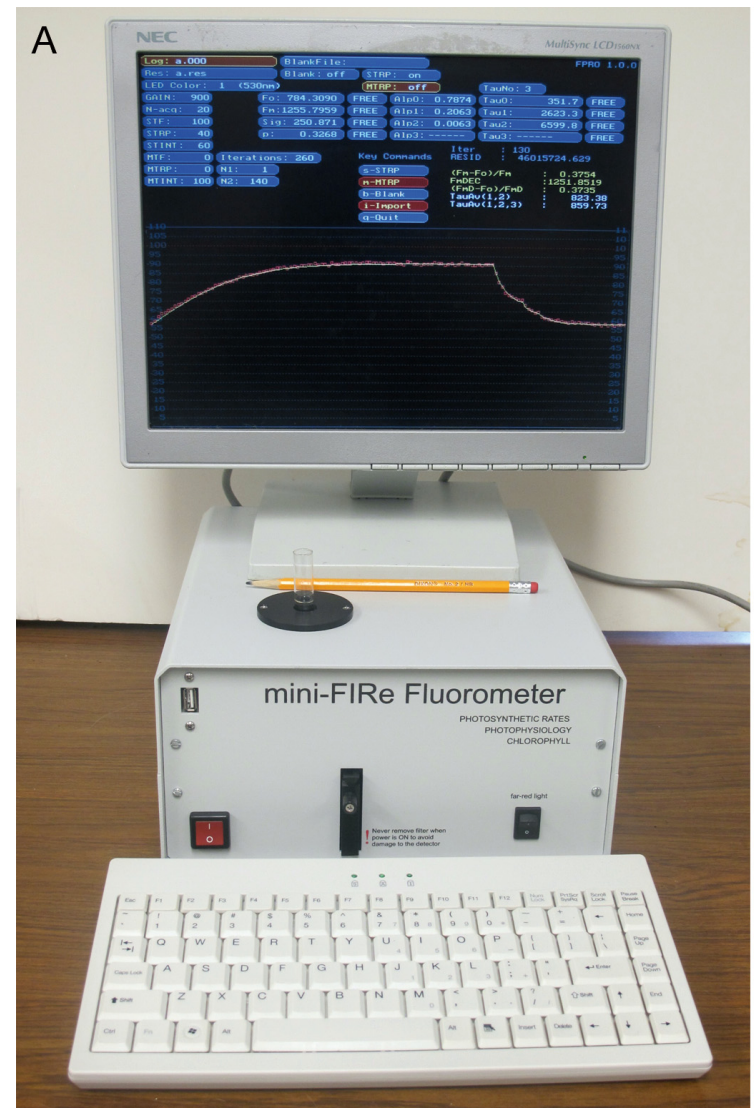

\section{MATERIALS AND METHODS}

\subsection{Mini-FIRe instrument and measurement protocols}

Variable fluorescence and photosynthetic characteristics were measured with a new miniaturized FIRe system (mini-FIRe) developed and manufactured by M. Gorbunov (Fig. 1A). This instrument is conceptually similar to previous FRR and FIRe systems (Gorbunov \& Falkowski 2005), but exhibits ca. 20-fold higher sensitivity and signal-to-noise ratio, compared to its predecessors. This extreme sensitivity is crucial for sampling in oligotrophic regions, which constitute $30 \%$ of the world oceans (Lin et al. 2016). The miniFIRe instrument also employs excitation at multiple wavelengths $(\lambda)$ that allows for selective excitation of different functional groups of phytoplankton, as well as for spectrally resolved measurements of functional absorption cross-sections of PSII, $\sigma_{\text {PSII }}(\lambda)$. The instrument used in this study employed 6 excitation channels $(435,450,470,500,530$, and $590 \mathrm{~nm}$, with $20 \mathrm{~nm}$ bandwidth for each channel) The peak excitation intensity in the sounding volume, i.e. the volume from which the signal is recorded, ranges from ca. 20000 to $25000 \mu \mathrm{mol}$ quanta $\mathrm{m}^{-2} \mathrm{~s}^{-1}$ for blue excitation channels $(435,450,470,500 \mathrm{~nm})$ and 40000 to $60000 \mu \mathrm{mol}$ quanta $\mathrm{m}^{-2} \mathrm{~s}^{-1}$ for green and orange excitation. These

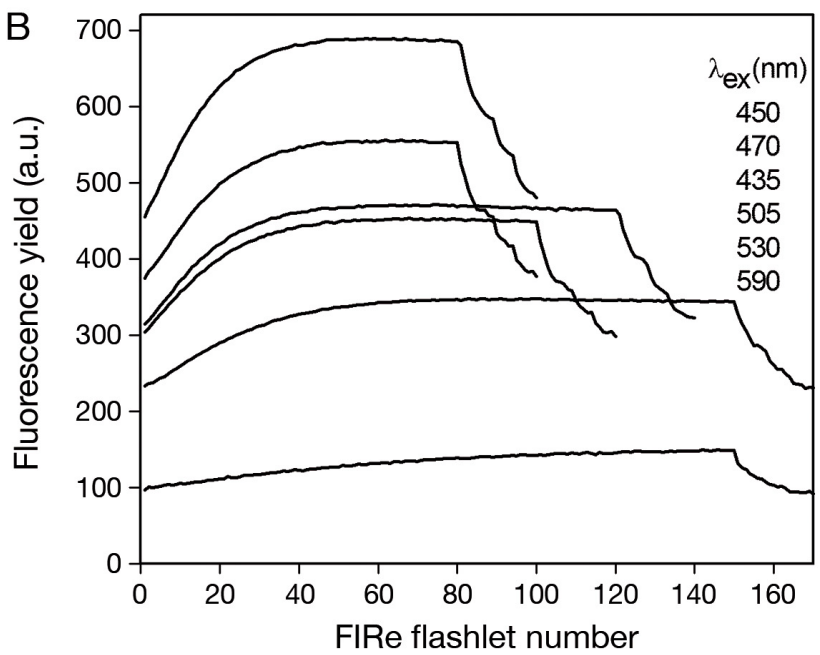

Fig. 1. (A) Multi-color fluorescence induction and relaxation instrument (mini-FIRe). (B) FIRe fluorescence profiles collected on a dilute sample of the marine diatom Phaeodactylum tricornutum, with chl a concentration of ca. $0.5 \mathrm{mg} \mathrm{m}^{-3}$. The induction part of the FIRe protocol was recorded for a single turnover flash (STF): $80 \mu \mathrm{s}$ (STF) for blue excitation and $150 \mu \mathrm{s}$ STF for green and orange excitation. The excitation wavelengths $\left(\lambda_{\text {ex }}\right)$ for FIRe traces (from top to bottom) are shown in the key. The relaxation part was recorded on a time scale from 0 to $200 \mathrm{~ms}$ (note the non-linear time scale for the relaxation part; Gorbunov \& Falkowski 2005). The quantum yield of photochemistry in PSII (i.e. photosynthetic efficiency) is deduced from the relative change in fluorescence yield $\left(F_{\mathrm{v}} / F_{\mathrm{m}}\right)$. The effective absorption cross-section of PSII $\left(\sigma_{\mathrm{PSII}}\right)$ is derived from the rate of fluorescence rise during the induction curve. The subsequent relaxation in fluorescence yield on micro- and millisecond time scales reflects the rates of photosynthetic electron transport delivered on the acceptor side of PSII 
higher excitation intensities from the green and orange light-emitting diodes (LEDs, Luxeon Rebel) are achieved by applying supra-optimal electric current (ca. $5 \times$ nominal values) in short pulses. The excitation intensities for all spectral channels are optimally adjusted to ensure closure of PSII reaction centers and saturation of fluorescence within ca. $100 \mu$ s (i.e. a single photosynthetic turnover) in each excitation channel (Fig. 1B), which is crucially important for accurate retrievals of functional cross-sections and quantum yields (Gorbunov et al. 1999).

The fluorescence signal is collected from a central, $8 \mathrm{~mm}$ diameter, portion of the sample volume, isolated by a red band pass interference filter (680 nm, with $20 \mathrm{~nm}$ bandwidth), and detected by an avalanche photodiode (SD630-70-72-500, Advanced Photonics). The voltage on the detector is automatically adjusted depending on ambient temperature to ensure a constant gain over a wide temperature range $\left(0\right.$ to $\left.30^{\circ} \mathrm{C}\right)$. A small portion of the excitation light is recorded by a PIN photodiode as a reference signal. By using an avalanche photodiode with high quantum efficiency (ca. $80 \%$ ) in the red spectral region and integrating the fluorescence signal in the analog mode, the instrument operates with an extremely high signal-to-noise ratio. The mini-FIRe instrument can accurately measure fluorescence signals in samples as low as $0.01 \mathrm{mg}$ chlorophyll $\mathrm{m}^{-3}$, effectively the lowest concentration of chlorophyll recorded anywhere in the upper ocean.

$\sigma_{\text {PSII }}$ is a product of the optical absorption cross-section of PSII (i.e. the size of the PSII antennae) and the quantum yield of photochemistry in PSII (Falkowski et al. 2004b). $\sigma_{\text {PSII }}$ is calculated from the rate of fluorescence rise during the single turnover flash (Fig. 1), as this rate is proportional to the product of $\sigma_{\mathrm{PSII}}$ and the photon flux density of excitation light (Gorbunov \& Falkowski 2005). Retrievals of $\sigma_{\text {PSII }}$ in absolute units $\left(\AA^{2}\right)$ require accurate calibration of the average photon flux density in the sounding volume (Gorbunov \& Falkowski 2005). Because the taxonomic analysis relies on quantifying small changes in the spectral shape, accurate calibration of all color channels is crucially important. Such calibration is conducted as part of the standard calibration procedure of the FIRe instrument.

\subsection{Strains and culture conditions}

The eukaryote algal cultures were grown at $18^{\circ} \mathrm{C}$ in $\mathrm{F} / 2$ medium in a 12:12 h light:dark cycle and white fluorescent light $\left(100 \mu \mathrm{mol}\right.$ photons $\left.\mathrm{m}^{-2} \mathrm{~s}^{-1}\right)$. These cultures included diatoms (Thalassiosira pseudonana
CCMP1335, Thalassiosira weissflogii CCMP1336, Chaetoceros muelleri CCMP1318, Navicula incerta UTEX 2044, Ditylum brightwellii CCMP358, Thalassiosira oceanica CCMP1005, Phaeodactylum tricornutum CCMP632, and Phaeodactylum tricornutum CCMP2559), dinoflagellates (Symbiodinium sp. CCMP832 and CCMP2467, Amphidinium carterae CCMP1314, and Prorocentrum micans CCMP1589), haptophytes (Emiliania huxleyi CCMP 373, CCMP 374, CCMP379, CCMP1516, and Gephyrocapsa oceanica AC396), and chlorophytes (Dunaliella tertiolecta CCMP1320, Chlorella vulgaris, Tetraselmis chuii, and Chlamydomonas reinhardtii). The cultures of cyanobacteria (Synechococcus sp. CCMP 1379, CCMP 2370 , and PCC 7002) were grown at $25^{\circ} \mathrm{C}$ in $\mathrm{F} / 2$ media. The cyanobacterium Arthrospira maxima was grown at $25^{\circ} \mathrm{C}$ in BG-11 media. Because cyanobacteria are known to exhibit strong chromatic adaptation in their pigment content and spectral properties, we grew cyanobacteria cultures under white and blue (central wavelength $455 \mathrm{~nm}$, half-bandwidth $30 \mathrm{~nm}$ ) light from a custom-built LED lamp. The white light was intended to simulate conditions in the upper ocean layer, while the blue light simulated the irradiance regime in open-ocean deep blue waters.

\subsection{Nutrient stress experiments}

Nutrient stress experiments were conducted on batch cultures growing in low-nitrogen media. First, cultures were pre-acclimated (for 4-5 d) to low-nitrogen $\mathrm{F} / 2$ medium, with $\left[\mathrm{NO}_{3}\right]$ of $10 \mu \mathrm{M}$, which is ca. 90 times lower than in normal F/2. This pre-acclimation allowed the cells to maintain maximum growth rates even in this low-nitrogen medium, although the cellular chlorophyll a ( $\mathrm{chl}$ a) content reduced several fold (a sign of acclimation to low-nitrogen conditions). We then monitored, in batch cultures, the slowly progressing nutrient stress, as the cells digested the remaining $\left[\mathrm{NO}_{3}\right]$ and the nitrogen concentration in the medium slowly reduced down to 0 within $48-72 \mathrm{~h}$. The chl a concentration in the samples during these experiments did not exceed 5-10 $\mathrm{mg} \mathrm{chl} \mathrm{m}^{-3}$ to ensure the slow development of nitrogen stress.

\subsection{Growth rate measurements}

Instantaneous growth rates $(\mu)$ were determined from an increase in cell density $\left(N\right.$, cells $\left.\mathrm{ml}^{-1}\right)$ over a short ( 4 to $6 \mathrm{~h}$ ) period of time $(\delta t)$, using an exponential growth equation: 


$$
\mu=\ln [N(t+\delta t) / N(t)] / \delta t
$$

Cell densities were recorded using a Beckman Coulter Multisizer 3 cell counter.

\subsection{Fluorescence spectroscopy}

Fluorescence excitation spectra were measured on a Jasco FP-8500 spectrofluorometer, by scanning the excitation wavelengths over the visible region (from 400 to $660 \mathrm{~nm}$ ) and recording chl a fluorescence emission at $690 \mathrm{~nm}$ (with $5 \mathrm{~nm}$ spectral bandwidth). In any conventional spectrofluorometer, the excitation intensities vary with wavelength, inevitably causing variations in the fraction of dynamically closed reaction centers and resulting in variations in the fluorescence quantum yield during the scan. As a consequence, the measured fluorescence excitation spectra may be severely distorted in photosynthetically active cells. To avoid this artifact, we treated all samples with 3-(3,4-dichlorophenyl)-1,1-dimethylurea (DCMU) and recorded fluorescence excitation spectra at the $F_{\mathrm{m}}$ level, with all reaction centers closed.

We should stress that, for the above reason, conducting measurements on DCMU-treated samples is the only way to correctly measure fluorescence excitation spectra in phytoplankton using conventional spectrofluorometers.

\subsection{Field studies}

Multi-spectral variable fluorescence data were collected during the annual Western Antarctic Peninsula Long Term Ecological Research (WAP LTER) cruise in the austral summer (January-February) of 2017 aboard the RV 'Laurence M. Gould.' The WAP LTER encompasses a grid spanning an area of $\sim 900 \times$ $200 \mathrm{~km}$ across the WAP shelf (Montes-Hugo et al. 2009). Samples were collected in Niskin bottles and kept at in situ temperature $\left(0\right.$ to $\left.1^{\circ} \mathrm{C}\right)$. During the day time, samples were acclimated at low light (ca. 5 $\mu \mathrm{mol}$ quanta $\mathrm{m}^{-2} \mathrm{~s}^{-1}$ ) for 30-60 min prior to measurements, to ensure recovery of variable fluorescence from non-photochemical quenching and photoinhibition. We should note that low-light (not dark) acclimation was crucial for recovery from photoinhibition, because this recovery is energetically driven by the $\Delta \mathrm{pH}$ gradient across the cellular thylakoid membrane produced by low-light exposure. Phytoplankton community structure was analyzed using microscopic analysis or HPLC (Montes-Hugo et al. 2009).

\subsection{Statistical analysis}

Data analyses were performed using the Python programming language and the 'scikit-learn' library. The K-means clustering algorithm was employed for the analysis of the spectra. The number of clusters was set to 4 based on a priori information on the number of phytoplankton functional groups. It was expected that due to significant variations between the spectra for different taxonomic groups, the cluster analysis would divide the dataset into 4 subsets. The clustering procedure yielded the centers of these 4 clusters (centroids), which were further compared to the basic spectra obtained by averaging the experimental spectra for each group. Based on the proximity of spectral band shapes, the 4 clusters were attributed to diatoms, dinoflagellates, haptophytes, and green algae.

\section{RESULTS}

\subsection{Spectral dependence of $\sigma_{\mathrm{PSII}}(\lambda)$ as a taxonomic index}

The proposed approach for taxonomic analysis relies on using the spectral dependences of $\sigma_{\text {PSII }}\left(\lambda_{\text {ex }}\right)$ and $F_{\mathrm{m}}\left(\lambda_{\mathrm{ex}}\right)$. We hypothesized that each taxonomic group exhibits its own phylum-specific spectra and that spectral variations within each group are smaller than the differences between different groups. To test this hypothesis, we analyzed multi-color FIRe measurements of the dependences $\sigma_{\mathrm{PSII}}\left(\lambda_{\mathrm{ex}}\right)$ and $F_{\mathrm{m}}\left(\lambda_{\text {ex }}\right)$ for a wide variety of eukaryote cultures.

Fig. 2 demonstrates the $\sigma_{\mathrm{PSII}}\left(\lambda_{\mathrm{ex}}\right)$ spectral dependences obtained for 4 major taxonomic groups of eukaryotic phytoplankton, including diatoms (7 cultures, Fig. 2A), dinoflagellates (3 cultures, Fig. 2B), green algae ( 4 cultures, Fig. 2C), and haptophytes (4 cultures, Fig. 2D).

Although the absolute values of $\sigma_{\text {PSII }}$ varied by a factor of ca. 2 within each taxonomic group (Fig. 2), the spectral shape of $\sigma_{\mathrm{PSII}}\left(\lambda_{\text {ex }}\right)$ was remarkably constrained within each group of eukaryotes. Fig. 3A shows the normalized $\sigma_{\mathrm{PSII}}\left(\lambda_{\mathrm{ex}}\right)$ spectra averaged over each group. We call these averaged normalized spectra the 'basic spectra' for each group. For each pairwise comparison between basic spectra, there is a wavelength region where the divergence between these groups exceeds the dispersion, suggesting that these dependences can potentially be used to discriminate between different groups. 


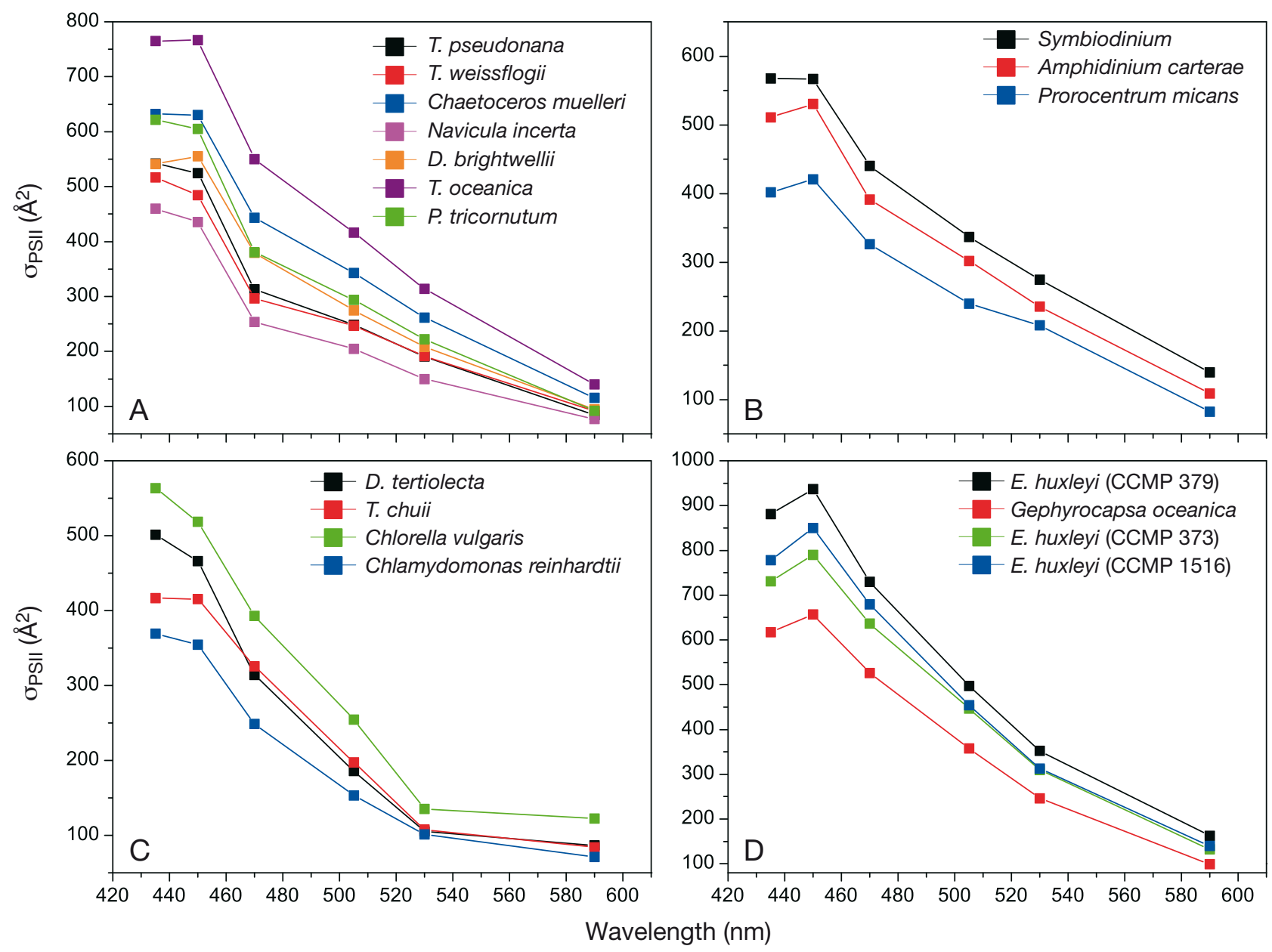

Fig. 2. Spectral dependence of the absorption cross-section $\sigma_{\text {PSII }}\left(\lambda_{\text {ex }}\right)$ for (A) diatoms, (B) dinoflagellates, (C) green algae, and (D) haptophytes. Each spectrum was averaged over several $(n=3-5)$ replicate measurements

We further compared the spectral dependencies $\sigma_{\mathrm{PSII}}\left(\lambda_{\mathrm{ex}}\right)$ of eukaryotes and prokaryotes. Fig. 4 shows $\sigma_{\mathrm{PSII}}\left(\lambda_{\mathrm{ex}}\right)$ for 4 cyanobacterial cultures, in comparison with the average $\sigma_{\mathrm{PSII}}\left(\lambda_{\text {ex }}\right)$ spectra for 4 major phylogenetic groups of eukaryotes. The cross-sections recorded at a single wavelength, $\sigma_{\text {PSII }}(450 \mathrm{~nm})$, were extremely small in cyanobacteria (Fig. 4)-several-fold smaller than in eukaryotes, consistent with previous observations (Suggett et al. 2009, Gorbunov et al. 2011). Moreover, in contrast to eukaryotes, the spectral shape of $\sigma_{\text {PSII }}$ varied dramatically among cyanobacterial species (Fig. 4), reflecting variations in phycobilin content and composition.

Chromatic adaptation to blue light decreased by $20-30 \% \sigma_{\text {PSII }}(590 \mathrm{~nm})$ relative to $\sigma_{\text {PSII }}$ in the bluegreen spectral region in Synechococcus spp. CCMP 1379 and PCC 7002. However, chromatic adaptation had virtually no effect on the spectral shape $\sigma_{\mathrm{PSII}}\left(\lambda_{\text {ex }}\right)$ in Synechococcus spp. CCMP 2370.

\subsection{Mathematical algorithm for using $\sigma_{\mathrm{PSII}}\left(\lambda_{\mathrm{ex}}\right)$ as a taxonomic index}

Based on the observed differences in the basic spectra of major phylogenetic groups of eukaryotes, we developed and tested the following mathematical algorithm for taxonomic identification.

First, we define the spectral slope $S_{i: j}$ for the basic spectra as:

$$
S_{i: j}=\left(S\left(\lambda_{i}\right)-S\left(\lambda_{j}\right)\right) /\left(\lambda_{i}-\lambda_{j}\right)
$$

where $S$ is $\sigma_{\text {PSII }}$ or $F_{\mathrm{m}}$ for the $\sigma_{\text {PSII }}$-based or $F_{\mathrm{m}^{-}}$ based algorithm, respectively; $i, j=435,450, \ldots$ 590 correspond to the 6 excitation wavelengths used in our instrument. Based on the $S_{i: j}$ values, the following classification procedure is proposed (Fig. 3B):

First, green algae are identified by a reduced value of $S_{530: 590}$, which is due to low absorption in the green spectral range $(530 \mathrm{~nm})$. 


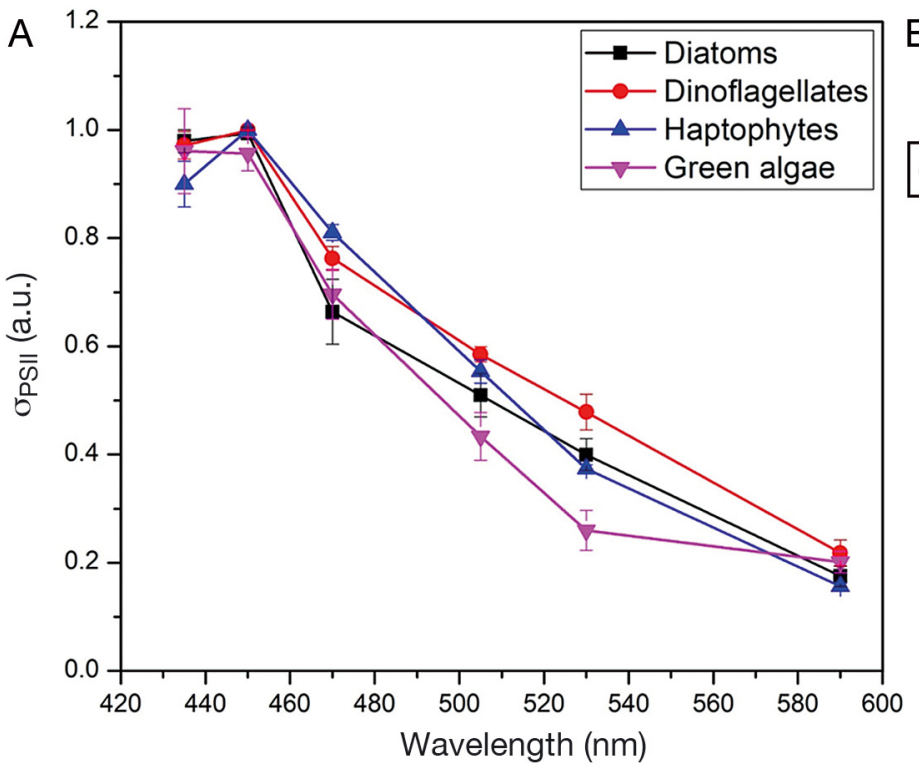

$B$

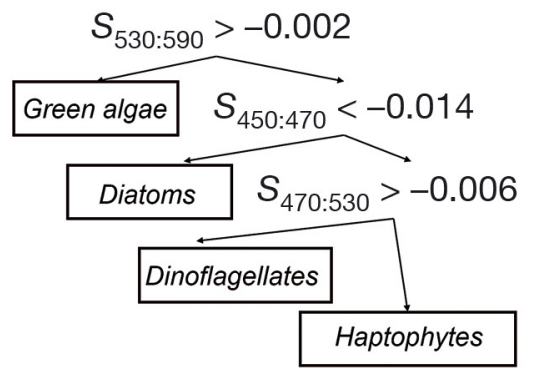

Fig. 3. (A) Normalized $\sigma_{\mathrm{PSII}}\left(\lambda_{\mathrm{ex}}\right)$ spectra (i.e. 'basic' spectra) of 4 different phylogenetic groups of eukaryotes (a.u.: arbitrary units; error bars are SD). (B) Schematic representation of the procedure used for phylogenetic group determination based on analysis of the $\sigma_{\mathrm{PSII}}\left(\lambda_{\mathrm{ex}}\right)$ spectral shape. Here, $S_{i: j}$ is the spectral slope obtained for the region of the $\sigma_{\mathrm{PSII}}\left(\lambda_{\text {ex }}\right)$ spectrum in the $\lambda_{\mathrm{i}}: \lambda_{\mathrm{j}}$ range (see Section 3.2 for details)

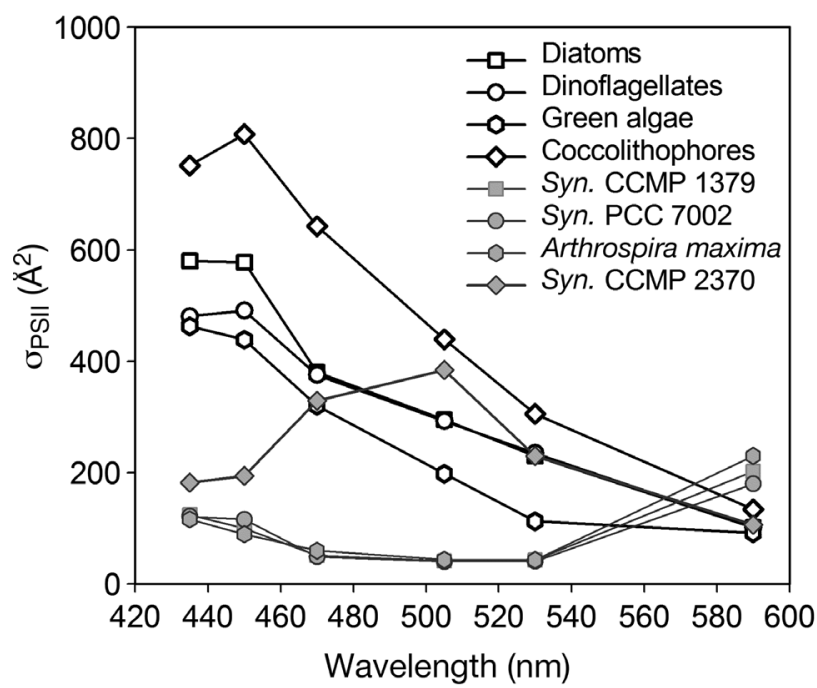

Fig. 4. Spectra $\sigma_{\mathrm{PSII}}\left(\lambda_{\text {ex }}\right)$ for selected cyanobacteria (gray symbols) and the averaged spectra $\sigma_{\mathrm{PSII}}\left(\lambda_{\mathrm{ex}}\right)$ for 4 major phylogenetic groups of eukaryotes (open symbols)

Second, diatoms are characterized by an enhanced value of $S_{450: 470}$, which is due to a steeper slope in the 450-470 range originating from an interplay between an increased chlorophyll $c$ content and a spectral dip caused by carotenoids.

Third, dinoflagellates and haptophytes are discriminated by the $S_{470: 530}$ value, whereby $\mathrm{S}_{470: 530}$ is smaller for haptophytes, as compared to dinoflagellates, due to differences in carotenoid content.

Application of this procedure to our dataset of $\sigma_{\mathrm{PSII}}(\lambda)$ for 18 eukaryote species yielded $90 \%$ accuracy of this identification algorithm. This result clearly suggests that the spectral variability of $\sigma_{\mathrm{PSII}}(\lambda)$ among different phylogenetic groups is sufficiently large to be used to functionally discriminate among the various phytoplankton taxa.

With the aim to achieve the maximum precision of the taxonomic identification algorithm, we used the entire dataset of spectral signatures for development and parameterization of this algorithm. At the same time, a common practice in validating such algorithms relies on splitting the data into 2 subsets ('training' and 'test') and using one of these subsets for development and another one for validation. For this reason, we conducted additional analyses by splitting our dataset into 2 independent 'training' and 'test' subsets. Our analysis revealed that by following this 'training-and-test' approach, the algorithm correctly identified $88 \%$ of the test data, which was very close to $90 \%$ correct identifications achieved for the above algorithm based on the entire dataset.

We further propose an alternative, more refined procedure for taxonomic identification, by introducing a 'proximity index' which characterizes proximity of a measured normalized spectrum $\sigma_{\text {PSII }}(\lambda)$ to the basic spectrum of a given phylogenetic group:

$$
n_{j}=\sqrt{\sum_{i}\left[S_{x}\left(\lambda_{i}\right)-B_{j}\left(\lambda_{i}\right)\right]^{2}}
$$

where $S_{x}$ is the analyzed spectrum and $B_{j}$ is the basic spectrum of the $j^{\text {th }}$ phylogenetic group. After calculating the discrepancies between the measured and all modeled basic spectra, the taxonomic identity of a sample is ascribed to the basic spectrum for which the $\Delta$ value is minimal. 
Application of this procedure to our dataset of $\sigma_{\text {PSII }}(\lambda)$ also yielded $90 \%$ correct identifications. Hence, our analysis clearly suggests that the spectral shape of $\sigma_{\text {PSII }}$ can be used as a taxonomic index, even for major phyla of the 'red' lineage of phytoplankton that have similar spectral properties.

To further evaluate the new multi-color FIRe approach for taxonomic analysis, we conducted an independent cluster analysis of the collected database of spectral signatures. This analysis automatically yielded 4 principal groups of spectra that correctly corresponded to the 4 phylogenetic groups used in our experiments (diatoms, haptophytes, dinoflagellates, and chlorophytes). The 4 basic spectra retrieved with this cluster analysis (Fig. 5B) were insignificantly different from the 4 basic spectra recorded experimentally (Fig. 5A).

\subsection{Effect of nutrient limitation on the spectral properties of $\sigma_{\mathrm{PSII}}$}

Phytoplankton photosynthesis in the global ocean is fundamentally limited by the availability of nutrients, such as nitrogen and iron (Lin et al. 2016). How does nutrient limitation affect the spectral properties of phytoplankton fluorescence, including $\sigma_{\mathrm{PSII}}\left(\lambda_{\text {ex }}\right)$ ? How might variations in the nutrient status affect the applications of the spectral-resolved variable fluorescence for taxonomic analysis?

To address these questions, we examined the effect of nitrogen limitation on $\sigma_{\mathrm{PSII}}\left(\lambda_{\mathrm{ex}}\right)$ in 2 model species, the diatom Phaeodactylum tricornutum and the chlorophyte Dunaliella tertiolecta. We quantified the

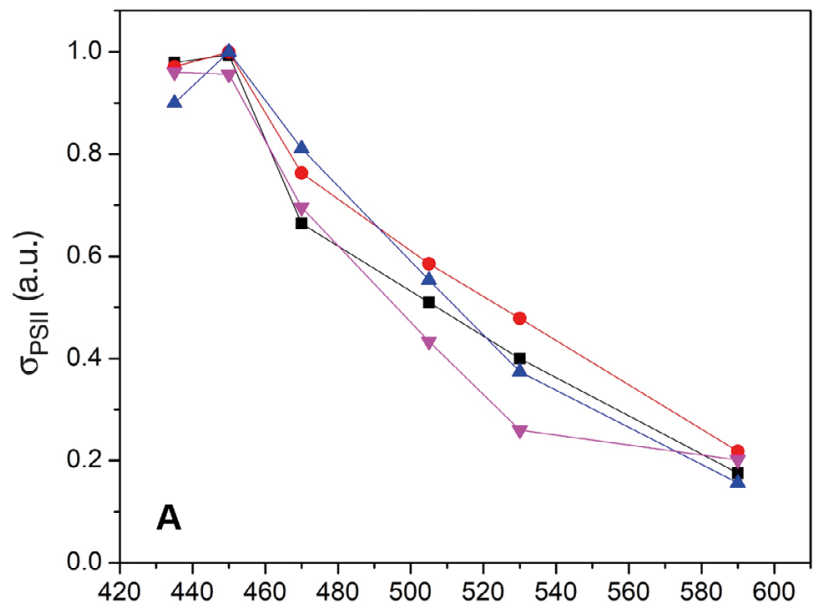

extent of nitrogen limitation from the reduction in the quantum yield of photochemistry in PSII $\left(F_{\mathrm{v}} / F_{\mathrm{m}}\right)$ and growth rates.

In D. tertiolecta, nitrogen limitation resulted in a characteristic decrease in growth rates, followed by a decrease in $F_{\mathrm{v}} / F_{\mathrm{m}}$ and an increase in $\sigma_{\mathrm{PSII}}$ (Fig. 6). Under severe nitrogen limitation, when the instantaneous growth rates reduced by $\sim 90 \%, F_{\mathrm{v}} / F_{\mathrm{m}}$ decreased by ca. $50 \%$ and $\sigma_{\text {PSII }}$ increased by $40 \%$ (Fig. 6). These changes in $F_{\mathrm{v}} / F_{\mathrm{m}}$ and $\sigma_{\mathrm{PSII}}$ are typical signatures of nutrient stress in phytoplankton (Kolber et al. 1988, Falkowski et al. 2004b). The decrease in $F_{\mathrm{v}} / F_{\mathrm{m}}$ indicates a reduction in the fraction of active PSII reaction centers. Thereby, the absorbed light energy can be transferred from inactive photosynthetic units to remaining active centers, thus increasing the effective absorption cross-section of these active centers. Also, a decrease in cellular pigment density under nutrient limitation leads to reduction in the package effect, further increasing the effective absorption cross-section.

Nitrogen limitation had no effect on the spectral shape of $\sigma_{\text {PSII }}(\lambda)$ (Fig. 6). For instance, changes in the normalized cross-sections, $\sigma_{\text {PSII }}\left(\lambda_{\text {ex }}\right) / \sigma_{\text {PSII }}$ max were not statistically significant ( $\mathrm{p}>0.5$ ) for all FIRe wavelengths, except $435 \mathrm{~nm}$. Nitrogen limitation resulted in a small $(3 \%)$, but statistically significant $(\mathrm{p}<0.05)$ decrease in the ratio $\sigma_{\mathrm{PSII}}(435) / \sigma_{\mathrm{PSII}}(450)$. The wavelength of $435 \mathrm{~nm}$ corresponds to the maximum of chl a absorption, and the relative decrease in $\sigma_{\mathrm{PSII}}(435)$ is indicative of a reduction in the cellular chl a content (chlorosis), which is typically observed under nitrogen and iron limitation (Kolber et al. 1988, Greene et al. 1991).

In $P$. tricornutum, nitrogen limitation also resulted in a characteristic decrease in the growth rates, fol-

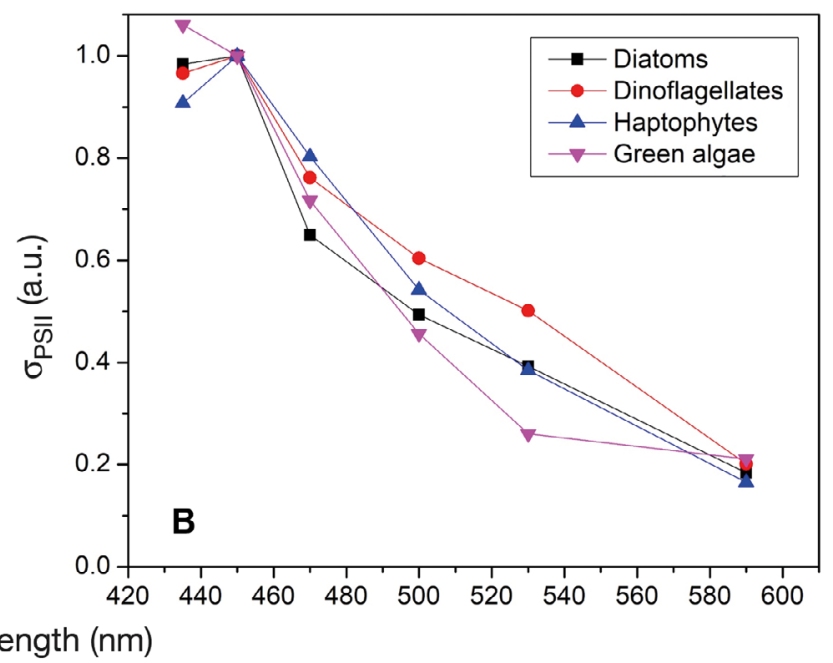

Fig. 5. Basic spectra $\sigma_{\mathrm{PSII}}\left(\lambda_{\text {ex }}\right)$ for 4 phylogenetic groups of eukaryotes, obtained by using 2 independent approaches. (A) Basic spectra obtained by averaging the experimental spectra for monocultures of each phylogenetic group and (B) modeled basic spectra obtained as a result of the cluster analysis of 66 spectra of monocultures 

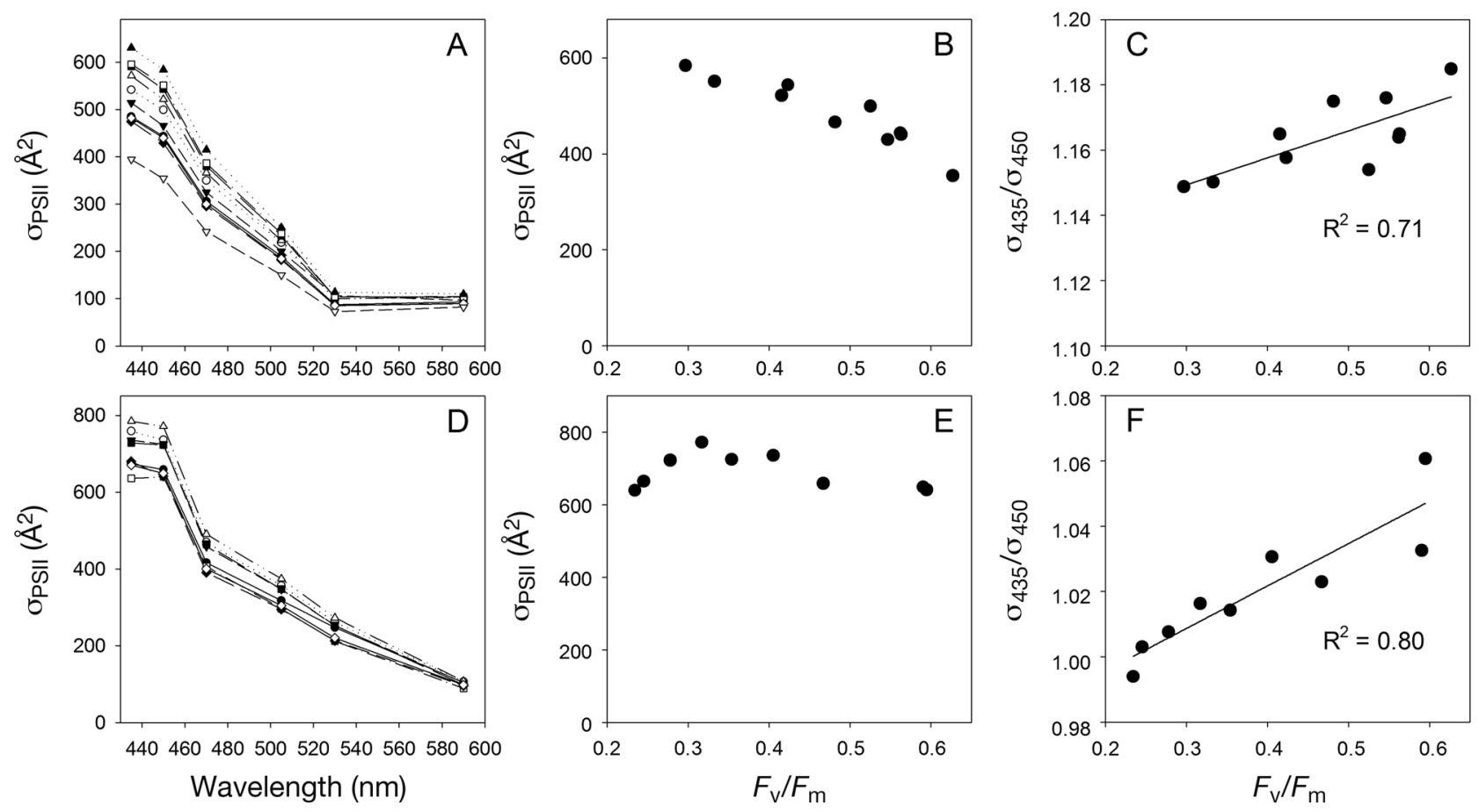

Fig. 6. Effect of nitrogen limitation on the spectral and photosynthetic characteristics of 2 model species: (A-C) the chlorophyte Dunaliella tertiolecta and (D-F) the diatom Phaeodactylum tricornutum

lowed by a decrease in $F_{\mathrm{v}} / F_{\mathrm{m}}$ and an increase in $\sigma_{\mathrm{PSII}}$ (Fig. 6D-F). However, the increase in $\sigma_{\mathrm{PSII}}$ was much smaller than in Dunaliella. Furthermore, under severe nitrogen limitation $\left(\mu<0.2 \mu_{\max }\right.$ and $F_{\mathrm{v}} / F_{\mathrm{m}}<$ $0.3), \sigma_{\mathrm{PSII}}$ decreased to the values typical of nutrientreplete cells (Fig. 6). The spectral shape of $\sigma_{\mathrm{PSII}}(\lambda)$ was not affected by nitrogen limitation for all wavelengths ( $p>0.5$ ), except $435 \mathrm{~nm}$ (Fig. 6). In P. tricornutum, the reduction in $\sigma_{\mathrm{PSII}}(435) / \sigma_{\mathrm{PSII}}(450)$ under severe nitrogen limitation was more pronounced than in Dunaliella (6 vs. $3 \%$ ).

\subsection{Comparing $\sigma_{\mathrm{PSII}}\left(\lambda_{\mathrm{ex}}\right)$ and $\boldsymbol{F}_{\mathrm{m}}\left(\lambda_{\mathrm{ex}}\right)$ as taxonomic indexes}

We further examined which of the spectral dependencies, $\sigma_{\mathrm{PSII}}\left(\lambda_{\text {ex }}\right)$ or $F_{\mathrm{m}}\left(\lambda_{\text {ex }}\right)$, is more accurate for taxonomic analysis. For this goal, we first reconstructed the basic spectra $F_{\mathrm{m}}(\lambda)$ for the 4 major phylogenetic groups of eukaryotes (Fig. 7). These basic spectra were defined using the same database that we used for the $\sigma_{\mathrm{PSII}}\left(\lambda_{\mathrm{ex}}\right)$ analysis above. We then applied the same mathematical procedure for $F_{\mathrm{m}}\left(\lambda_{\mathrm{ex}}\right)$ analysis as we did for $\sigma_{\mathrm{PSII}}\left(\lambda_{\mathrm{ex}}\right)$. Surprisingly, this $F_{\mathrm{m}}$ based analysis yielded only $76 \%$ correct identifications, as compared to $90 \%$ for the $\sigma_{\mathrm{PSII}}$-based analysis.
Next, we applied hyper-spectral fluorescence excitation spectra measured using a conventional fluorometer for taxonomic identification. Fig. 8 demonstrates the normalized averaged fluorescence excitation spectra for 4 major groups of eukaryotes.

Using the same procedure based on evaluation of proximity to basic spectra (Eq. 3), we obtained $89 \%$

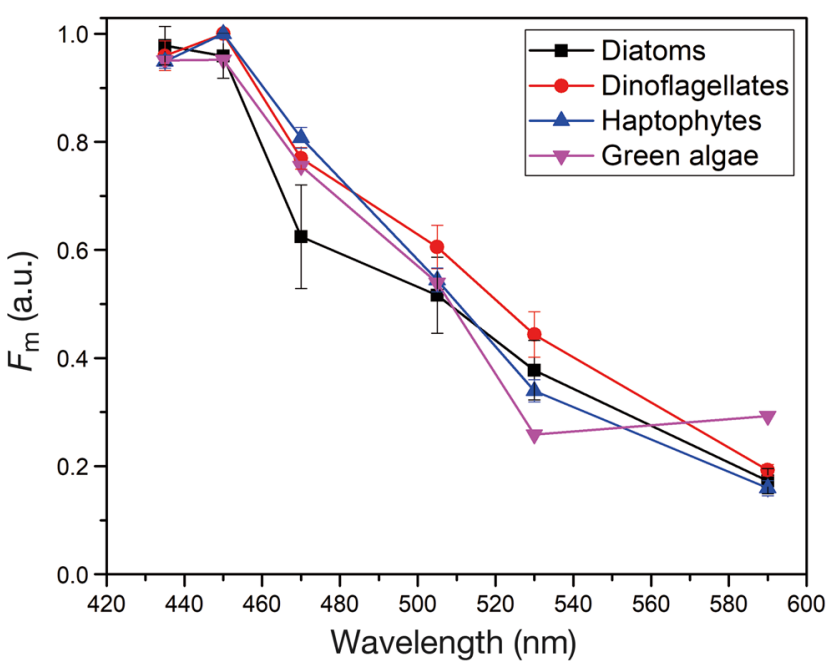

Fig. 7. Normalized $F_{\mathrm{m}}\left(\lambda_{\mathrm{ex}}\right)$ spectral dependences for different phylogenetic groups. For each phylogenetic group, averaging was performed over all individual spectra of monocultures, normalized to the maximum value of $F_{\mathrm{m}}$. Error bars are SD 


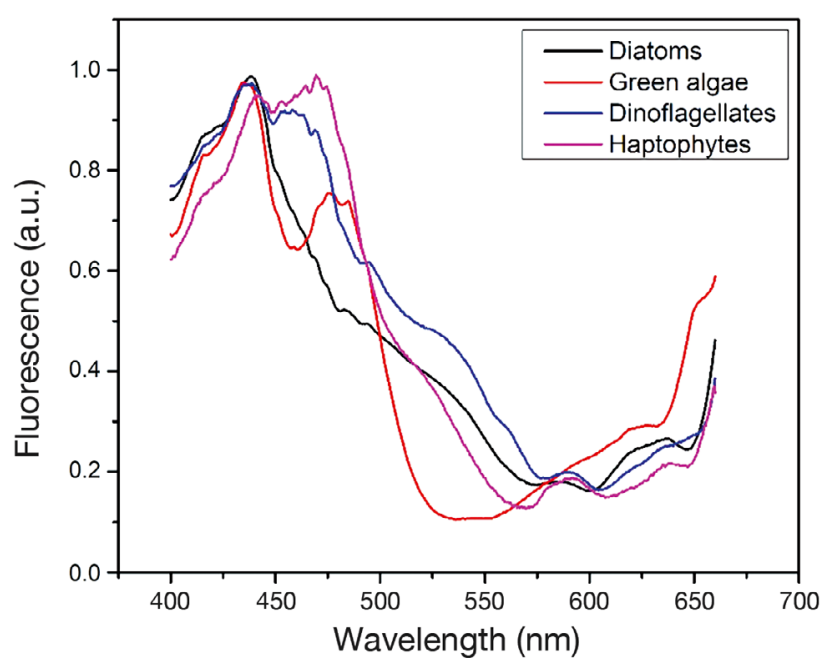

Fig. 8. Normalized averaged fluorescence excitation spectra for different phylogenetic groups, measured using a standard hyper-spectral fluorometer

correct identifications. This number is very close to that obtained using the $\sigma_{\mathrm{PSII}}\left(\lambda_{\mathrm{ex}}\right)$ algorithm above. At the same time, it clearly shows that the use of 6 excitation wavelengths was not sufficient for accurate identification using conventional fluorescence excitation spectra (e.g. $F_{\mathrm{m}}\left(\lambda_{\text {ex }}\right)$ ).

\subsection{Field evaluation}

Field validation of the proposed approach was performed using a dataset collected during the WAP LTER cruise off the WAP. The proximity indexes to the basic spectra of major functional groups were calculated for 150 samples. This analysis resulted in $95 \%$ of spectra being identified as diatoms, which was in good agreement with the microscopic examination of samples. Fig. 9 shows the average normalized $\sigma_{\mathrm{PSII}}(\lambda)$ spectrum obtained for the Antarctic communities, as well as the basic spectrum for diatoms obtained from model experiments on diatoms (Figs. $2 \& 3$ ). Our taxonomic algorithm identified a small fraction $(5 \%)$ of field spectra as being assigned to dinoflagellates. These false identifications were for deep-water (>60 m) samples with low chlorophyll content and thus low signal-to-noise ratio and with an elevated amount of fluorescent pigment degradation products.

\section{DISCUSSION}

Our research has revealed that, in spite of variability in pigment composition, $\sigma_{\mathrm{PSII}}\left(\lambda_{\text {ex }}\right)$ is remarkably

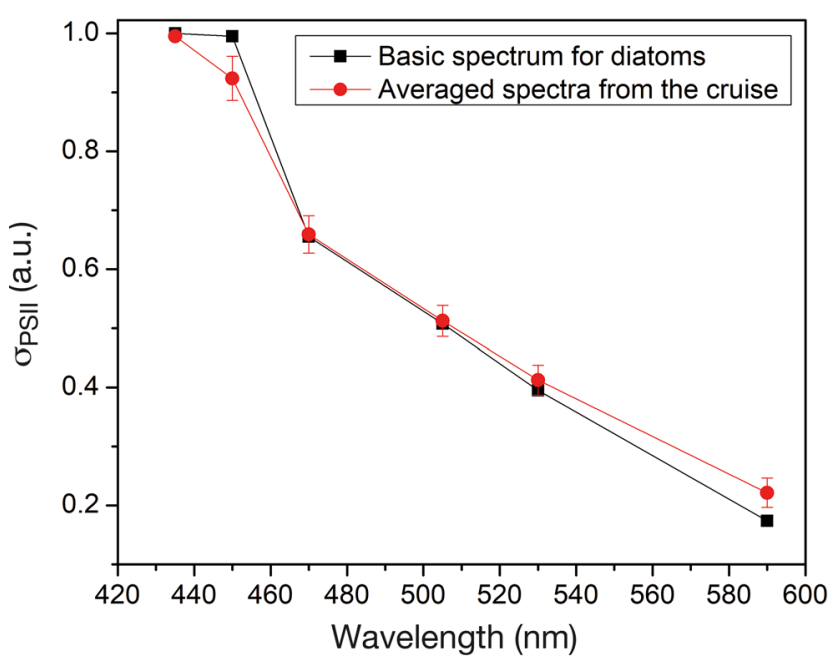

Fig. 9. Averaged normalized $\sigma_{\mathrm{PSII}}\left(\lambda_{\text {ex }}\right)$ spectra for the Antarctic phytoplankton (red line), based on 150 samples collected off the Western Antarctic Peninsula. Black line corresponds to the basic spectrum of diatoms used for cluster analysis

constrained within each major taxonomic group of eukaryotic phytoplankton, including diatoms, haptophytes, dinoflagellates, and chlorophytes (Fig. 2A). Variability in $\sigma_{\mathrm{PSII}}\left(\lambda_{\text {ex }}\right)$ within each group was significantly smaller than the difference between individual groups, thus providing the basis for taxonomic identification algorithms. Although the big difference in spectral properties (i.e. color) between the 'green' and 'red' lineages of eukaryotic algae is well known, the discovered differences between the 3 major phyla of the 'red' lineage (diatoms, haptophytes, dinoflagellates) were unexpected. These ecologically dominant eukaryote groups exhibit similar spectral properties and remain extremely challenging for spectroscopic identification. Our results suggest that the multi-color variable fluorescence analysis offers a promising tool for the combined physiological and taxonomic analysis, including identification of 3 major phyla within the 'red' lineage of phytoplankton.

The absolute values of the cross-sections of PSII (e.g. $\sigma_{\text {PSII }}$ measured at a single wavelength, i.e. $450 \mathrm{~nm}$ ) vary 4 - to 5 -fold among species (Figs. $3 \& 4$; and see Suggett et al. 2009). In natural phytoplankton communities, changes in $\sigma_{\text {PSII }}$ often reflect changes in the community structure and cell size distribution (Gorbunov et al. 1999). For instance, due to a stronger pigment packaging effect, larger cells tend to have a smaller effective $\sigma_{\text {PSII }}$ compared to small cells (Gorbunov et al. 1999). Therefore, the magnitude of $\sigma_{\text {PSII }}$ at one excitation wavelength may carry information about taxonomic identity (Suggett 
et al. 2009). Our laboratory research supports this hypothesis, to a first approximation. Among eukaryotes, the highest $\sigma_{\mathrm{PSI}}$ values were observed in haptophytes and diatoms, and the lowest values in green algae and dinoflagellates (Fig. 2). In cyanobacteria, $\sigma_{\text {PSII }}(450 \mathrm{~nm})$ values were extremely small (Fig. 4): several-fold smaller than in eukaryotes, consistent with previous observations (Suggett et al. 2009, Gorbunov et al. 2011, Simis et al. 2012). Moreover, the spectral band shape of $\sigma_{\text {PSII }}$ for cyanobacteria is dramatically different from that of eukaryotes and varies dramatically among cyanobacteria species (Fig. 4; and see Simis et al. 2012). At the same time, big variations in $\sigma_{\text {PSII }}$ values were observed within each functional group of both cyanobacteria and eukaryotes (Figs. 3 \& 4). Also, changes in environmental conditions, such as nutrient limitation, have a profound effect on $\sigma_{\text {PSII }}$ (Fig. 6; and see Kolber et al. 1988, Greene et al. 1991). These results clearly suggest that the absolute values of $\sigma_{\text {PSII }}$ at one excitation wavelength are not unambiguously indicative of taxonomic identity.

Many prokaryotic phytoplankton, such as cyanobacteria, and some eukaryotes (cryptophytes) can be identified using spectroscopic analysis, based on characteristic orange fluorescence emission from their unique accessory pigments, phycobolins (phycoerythrins, phycocyanin, and allophycocyanin) (Chisholm et al. 1988, Olson et al. 1991, Lantoine \& Neveux 1997, Chekalyuk \& Hafez 2008). This fluorescence emission is spectrally separated from chl a fluorescence, although allophycocyanin fluorescence closely overlaps with that of chl a. All other eukaryotes only emit chl a fluorescence (e.g. Falkowski \& Raven 2007). This chl a emission band is virtually identical in all organisms, which makes fluorescence emission spectroscopy unsuitable for taxonomic analysis of major groups of eukaryotes.

Based on the analysis of spectral properties and physiological characteristics, we have developed and tested an operational protocol for phytoplankton taxonomic identification, based on the proximity of measured spectra to basic (i.e. average) spectra of phylogenetic groups. We have tested this protocol on the collected database of 25 species. As much as $90 \%$ of the experimental spectra of eukaryotic cultures were correctly classified, clearly suggesting a high predictive capability of this approach. Additional evidence for the principal difference in the basic spectra between the taxonomic groups comes from our cluster analysis of the collected database that in its main components was very similar to the experimentally obtained basic spectra.
In addition to the spectral shape of $\sigma_{\mathrm{PSII}}\left(\lambda_{\text {ex }}\right)$, we also examined the spectral shape of chl a fluorescence yields, $F_{\mathrm{m}}\left(\lambda_{\mathrm{ex}}\right)$, as a potential taxonomic proxy. The shape of $F_{\mathrm{m}}\left(\lambda_{\text {ex }}\right)$ reflects the classical chl a fluorescence excitation spectra (Lakowicz 2006). To a first approximation, the excitation spectra $F_{\mathrm{m}}\left(\lambda_{\mathrm{ex}}\right)$ should be virtually identical to $\sigma_{\mathrm{PSII}}\left(\lambda_{\mathrm{ex}}\right)$ because both variables are determined by the flux of excitons to the PSII core. Surprisingly, our research revealed that $\sigma_{\mathrm{PSII}}\left(\lambda_{\text {ex }}\right)$ is a better and more specific proxy for taxonomic analysis. For instance, the $\sigma_{\text {PSII }}$-based algorithm correctly identified $90 \%$ of experimental data, while the $F_{\mathrm{m}}$-based algorithm only identified $77 \%$.

The difference in the spectral shape between $\sigma_{\text {PSII }}\left(\lambda_{\text {ex }}\right)$ and $F_{\text {m }}\left(\lambda_{\text {ex }}\right)$ may be caused by the following: First, in intact cells, high intracellular density of photosynthetic pigments (i.e. the 'pigment packaging effect,' Geider \& Osborne 1987, Berner et al. 1989) decreases the effective intensity of incident light within the cell, thus affecting both $\sigma_{\mathrm{PSII}}$ and $F_{\mathrm{m}}$. In addition, the pigment packaging leads to re-absorption of emitted fluorescence, further reducing the measured $F_{\mathrm{m}}$, but not $\sigma_{\mathrm{PSII}}$. Therefore, the pigment packaging has a stronger effect on $F_{\mathrm{m}}$ than on $\sigma_{\mathrm{PSII}}$. Variations in pigment packaging are most pronounced in large cells and introduce errors into the $F_{\mathrm{m}}$-based algorithm. Second, measurements of fluorescence yields (both $F_{\mathrm{o}}$ and $F_{\mathrm{m}}$, but not $F_{\mathrm{v}}$ ) are sensitive to artifacts of blank correction (Bibby et al. 2008). Because $\sigma_{\mathrm{PSI}}$ is calculated from the variable fluorescence component (Falkowski et al. 2004b), $\sigma_{\mathrm{PSII}}\left(\lambda_{\text {ex }}\right)$ is not subject to errors in blank correction, at least in FIRe instruments.

Our approach has been implemented on the basis of a novel mini-FIRe system, which allows for simultaneous measurements of photophysiological parameters and spectrally-resolved fluorescence yields, $F_{\mathrm{o}}\left(\lambda_{\mathrm{ex}}\right)$ and $F_{\mathrm{m}}\left(\lambda_{\mathrm{ex}}\right)$, and $\sigma_{\mathrm{PSII}}\left(\lambda_{\mathrm{ex}}\right)$. The physiological parameters are used for assessment of photosynthetic rates, including primary productivity (Kolber \& Falkowski 1993, Hughes et al. 2018a, Ko et al. 2019), and the impact of environmental factors and stressors (Falkowski et al. 2004b, Gorbunov \& Falkowski 2005), while spectrally-resolved fluorescence yields are for taxonomic analysis. The miniFIRe system operates in 2 modes, including discrete sample analysis and a fully automatic on-line mode, using a flow-through cuvette with an anti-fouling cleaning system. These capabilities, in combination with extreme sensitivity, offer new prospects for automated monitoring of phytoplankton communities and primary production in the global ocean. 
The combination of photophysiological parameters and taxonomic proxies from the multi-color FIRe measurements may have important implications for improving fluorescence-based methods to measure primary production. The starting point in these methods is the measurement of photosynthetic electron transport rates (Kolber \& Falkowski 1993). The conversion of these rates to the rates of carbon fixation relies on the knowledge of electron requirements for carbon fixation (Kolber \& Falkowski 1993, Lawrenz et al. 2013). Extensive laboratory and field studies have revealed that the electron requirements in natural phytoplankton communities are controlled by the extent of nutrient limitation and also may vary with taxonomy (Lawrenz et al. 2013, Zhu et al. 2017, Hughes et al. 2018b, Ko et al. 2019). The multi-color variable fluorescence technique might provide crucial information about these 2 controls of the electron requirement for carbon fixation.

Although quantitative taxonomic analysis of natural communities using bulk fluorescence signals is still challenging and merits further research and development effort, our results clearly suggest that such analysis can be conducted, with high accuracy, on the level of a single species, for example using single-cell cytometric analysis. This result offers new opportunities for high-throughput cytometric analysis using multi-color fluorometry. Methodology and operational instrumentation for single-cell measurements of variable fluorescence have been developed (Gorbunov et al. 1999, Olson et al. 2000). Incorporation of multi-color protocols to such instrumentation would provide unprecedented capabilities for combined physiological and taxonomic analysis of natural phytoplankton communities in the ocean.

Acknowledgements. This research was supported by NASA Ocean Biology and Biogeochemistry Program (grants NNX16AT54G and 80NSSC18K1416 to M.Y.G. and P.G.F.) and the Russian Foundation of Fundamental Research (grants 18-35-00643 and 18-05-00941). We thank Jonathan Sherman and Oscar Schofield for assistance with field data collection, Kevin Wyman for suggestions on the manuscript, and the anonymous reviewers and the editor for constructive comments.

\section{LITERATURE CITED}

Babin M, Cullen JJ, Roesler CS, Donaghay PL and others (2005) New approaches and technologies for observing harmful algal blooms. Oceanography 18:210-227

Behrenfeld MJ, Bale AJ, Kolber ZS, Aiken J, Falkowski PG (1996) Confirmation of iron limitation of phytoplankton photosynthesis in the equatorial Pacific Ocean. Nature 383:508-511
Berner T, Dubinsky Z, Wyman K, Falkowski PG (1989) Photoadaptation and the 'package' effect in Dunaliella tertiolecta. J Phycol 25:70-78

Bibby TS, Gorbunov MY, Wyman KW, Falkowski PG (2008) Photosynthetic community responses to upwelling in mesoscale eddies in the subtropical North Atlantic and Pacific Oceans. Deep Sea Res II 55:1310-1320

Bidigare RR, Van Heukelem L, Trees CC (2005) Analysis of algal pigments by high-performance liquid chromatography. In: Anderson RA (ed) Algal culturing techniques. Academic Press, Burlington, MA, p 327-345

Chekalyuk A, Hafez M (2008) Advanced laser fluorometry of natural aquatic environments. Limnol Oceanogr Methods 6:591-609

* Chisholm SW, Olson RJ, Zettler ER, Goericke R, Waterbury J, Welschmeyer N (1988) A novel free-living prochlorophyte abundant in the oceanic euphotic zone. Nature 334:340-343

Claustre H (1994) The trophic status of various oceanic provinces as revealed by phytoplankton pigment signatures. Limnol Oceanogr 39:1206-1210

* Coale KH, Johnson KS, Chavez FP, Buesseler KO and others (2004) Southern ocean iron enrichment experiment: carbon cycling in high- and low-Si waters. Science 304: 408-414

Davis CS, Hu Q, Gallager SM, Tang X, Ashjian CJ (2004) Real-time observation of taxa-specific plankton distributions: an optical sampling method. Mar Ecol Prog Ser 284:77-96

Davis CS, Thwaites FT, Gallager SM, Hu Q (2005) A threeaxis fast-tow digital Video Plankton Recorder for rapid surveys of plankton taxa and hydrography. Limnol Oceanogr Methods 3:59-74

*Delwiche CF (1999) Tracing the thread of plastid diversity through the tapestry of life. Am Nat 154:S164-S177

Falkowski PG, Raven JA (2007) Aquatic photosynthesis, $2^{\text {nd }}$ edn. Princeton University Press, Princeton, NJ

Falkowski PG, Katz ME, Knoll AH, Quigg A, Raven JA, Schofield O, Taylor FJR (2004a) The evolution of modern eukaryotic phytoplankton. Science 305:354-360

Falkowski PG, Koblizek M, Gorbunov M, Kolber Z (2004b) Development and application of variable chlorophyll fluorescence techniques in marine ecosystems. In: Papageorgiou C, Govingjee (eds) Chlorophyll a fluorescence: a signature of photosynthesis. Springer, Dordrecht, p $757-778$

Field CB, Behrenfeld MJ, Randerson JT, Falkowski P (1998) Primary production of the biosphere: integrating terrestrial and oceanic components. Science 281:237-240

*Geider RJ, Osborne BA (1987) Light absorption by a marine diatom: experimental observations and theoretical calculations of the package effect in a small Thalassiosira species. Mar Biol 96:299-308

Gorbunov MY, Falkowski PG (2005) Fluorescence induction and relaxation (FIRe) technique and instrumentation for monitoring photosynthetic processes and primary production in aquatic ecosystems. In: van der Est A, Bruce D (eds) Photosynthesis: fundamental aspects to global perspectives. Proc $13^{\text {th }}$ Int Congress of Photosynthesis, Montreal, Aug 29 - Sep 3, 2004. Allen Press, Montreal, p 1029-1031

Gorbunov MY, Kolber Z, Falkowski PG (1999) Measuring photosynthetic parameters in individual algal cells by Fast Repetition Rate fluorometry. Photosynth Res 62: 141-153 
Gorbunov MY, Kuzminov FI, Fadeev VV, Kim JD, Falkowski PG (2011) A kinetic model of non-photochemical quenching in cyanobacteria. Biochim Biophys Acta Bioenerg 1807:1591-1599

Greene RM, Geider RJ, Falkowski PG (1991) Effect of iron limitation on photosynthesis in a marine diatom. Limnol Oceanogr 36:1772-1782

Hughes DJ, Campbell DA, Doblin MA, Kromkamp JC and others (2018a) Roadmaps and detours: active chlorophyll- $a$ assessments of primary productivity across marine and freshwater systems. Environ Sci Technol 52: 12039-12054

Hughes DJ, Varkey D, Doblin MA, Ingleton T and others (2018b) Impact of nitrogen availability upon the electron requirement for carbon fixation in Australian coastal phytoplankton communities. Limnol Oceanogr 63: 1891-1910

*Ko E, Park J, Gorbunov MY, Yoo S (2019) Uncertainties in variable fluorescence and ${ }^{14} \mathrm{C}$ methods to estimate primary production: a case study in the coastal waters off the Korean peninsula. Mar Ecol Prog Ser 627:13-31

Kolber Z, Falkowski PG (1993) Use of active fluorescence to estimate phytoplankton photosynthesis in situ. Limnol Oceanogr 38:1646-1665

Kolber Z, Zehr J, Falkowski P (1988) Effects of growth irradiance and nitrogen limitation on photosynthetic energy conversion in photosystem II. Plant Physiol 88:923-929

Kolber ZS, Prasil O, Falkowski PG (1998) Measurements of variable chlorophyll fluorescence using fast repetition rate techniques: defining methodology and experimental protocols. Biochim Biophys Acta Bioenerg 1367:88-106

Lakowicz JR (2006) Principles of fluorescence spectroscopy, $3^{\text {rd }}$ edn. Springer Science+Business Media, New York, NY

Lantoine F, Neveux J (1997) Spatial and seasonal variations in abundance and spectral characteristics of phycoerythrins in the tropical northeastern Atlantic Ocean. Deep Sea Res I 44:223-246

*awrenz E, Silsbe G, Capuzzo E, Ylöstalo P and others (2013) Predicting the electron requirement for carbon fixation in seas and oceans. PLOS ONE 8:e58137

Li WKW (1997) Cytometric diversity in marine ultraphytoplankton. Limnol Oceanogr 42:874-880

Lin H, Kuzminov FI, Park J, Lee SH, Falkowski PG, Gorbunov MY (2016) The fate of photons absorbed by phytoplankton in the global ocean. Science 351:264-267

MacIntyre HL, Lawrenz E, Richardson TL (2010) Taxonomic discrimination of phytoplankton by spectral fluorescence. In: Suggett DJ, Prasil O, Borowitzka MA (eds) Chlorophyll a fluorescence measurements in aquatic sciences: methods and applications. Springer, Dordrecht, p 129-169

Mackey MD, Mackey DJ, Higgins HW, Wright SW (1996) CHEMTAX - a program for estimating class abundances from chemical markers: application to HPLC measurements of phytoplankton. Mar Ecol Prog Ser 144:265-283

Montes-Hugo M, Doney SC, Ducklow HW, Fraser W, Martinson D, Stammerjohn SE, Schofield O (2009) Recent changes in phytoplankton communities associated with rapid regional climate change along the Western Antarctic Peninsula. Science 323:1470-1473

Oldham PB, Warner IM (1987) Analysis of natural phytoplankton populations by pattern-recognition of twodimensional fluorescence spectra. Spectrosc Lett 20: 391-413
Olson RJ, Sosik HM (2007) A submersible imaging-in-flow instrument to analyze nano- and microplankton: imaging FlowCytobot. Limnol Oceanogr Methods 5:195-203

Olson RJ, Zettler ER, Chisholm SW, Dusenberry JA (1991) Advances in oceanography through flow cytometry. In: Demers S (ed) Particle analysis in oceanography. NATO ASI Ser (Ser G Ecol Sci) Vol 27. Springer, Berlin, p 351-399

Olson RJ, Sosik HM, Chekalyuk AM, Shalapyonok A (2000) Effects of iron enrichment on phytoplankton in the Southern Ocean during late summer: active fluorescence and flow cytometric analyses. Deep Sea Res II 47: 3181-3200

* Olson RJ, Shalapyonok A, Sosik HM (2003) An automated submersible flow cytometer for analyzing pico- and nanophytoplankton: FlowCytobot. Deep Sea Res I 50: 301-315

Richardson TL, Lawrenz E, Pinckney JL, Guajardo RC, Walker EA, Paerl HW, MacIntyre HL (2010) Spectral fluorometric characterization of phytoplankton community composition using the algae online analyser. Water Res 44:2461-2472

Sathyendranath S, Stuart V, Nair A, Oka K and others (2009) Carbon-to-chlorophyll ratio and growth rate of phytoplankton in the sea. Mar Ecol Prog Ser 383:73-84

Seppälä J, Balode M (1997) The use of spectral fluorescence methods to detect changes in the phytoplankton community. Hydrobiologia 363:207-217

Sieracki CK, Sieracki ME, Yentsch CS (1998) An imagingin-flow system for automated analysis of marine microplankton. Mar Ecol Prog Ser 168:285-296

* Simis SGH, Huot Y, Babin M, Seppälä J, Metsamaa L (2012) Optimization of variable fluorescence measurements of phytoplankton communities with cyanobacteria. Photosynth Res 112:13-30

Sosik HM, Olson RJ (2007) Automated taxonomic classification of phytoplankton sampled with imaging-in-flow cytometry. Limnol Oceanogr Methods 5:204-216

Sosik HM, Olson RJ, Neubert MG, Solow AR (2003) Growth rates of coastal phytoplankton from time-series measurements with a submersible flow cytometer. Limnol Oceanogr 48:1756-1765

Suggett DJ, Moore CM, Hickman AE, Geider RJ (2009) Interpretation of fast repetition rate (FRR) fluorescence: signatures of phytoplankton community structure versus physiological state. Mar Ecol Prog Ser 376:1-19

* Uitz J, Claustre H, Morel A, Hooker SB (2006) Vertical distribution of phytoplankton communities in open ocean: an assessment based on surface chlorophyll. J Geophys Res 111:C08005

Uitz J, Huot Y, Bruyant F, Babin M, Claustre H (2008) Relating phytoplankton photophysiological properties to community structure on large scales. Limnol Oceanogr 53: $614-630$

* Yentsch CS, Phinney DA (1984) Fluorescence spectral signatures for studies of marine phytoplankton. Adv Chem Ser 209:259-274

Yentsch CS, Yentsch CM (1979) Fluorescence spectral signatures - characterization of phytoplankton populations by the use of excitation and emission spectra. J Mar Res 37:471-483

Zhu Y, Ishizaka J, Tripathy SC, Wang S and others (2017) Relationship between light, community composition and the electron requirement for carbon fixation in natural phytoplankton. Mar Ecol Prog Ser 580:83-100 